\title{
Salivary Gland Acinic Cell Carcinoma
}

National Cancer Institute

\section{Source}

National Cancer Institute. Salivary Gland Acinic Cell Carcinoma. NCI Thesaurus. Code C8013.

A carcinoma of the salivary gland characterized by serous acinar cell differentiation. The vast majority of cases occur in the parotid gland. It usually presents as a slowly enlarging mass. A minority of patients experience pain. It may recur or metastasize. Multiple recurrences and metastasis to cervical lymph nodes are usually associated with a poor prognosis. 